\title{
A Novel Metric for the Evaluation of IDSs Effectiveness
}

\author{
Khalid Nasr ${ }^{1}$ and Anas Abou El Kalam ${ }^{2}$ \\ ${ }^{1}$ Electrical Eng. Dept., Faculty of Engineering, Minia University, Egypt \\ khalid.nasr79@hotmail.com \\ ${ }^{2}$ OSCARS Laboratory, UCA/ENSA of Marrakesh, Morocco \\ anas@ensa.ac.ma
}

\begin{abstract}
Nowadays intrusion detection system (IDS) has a considerable attention as a crucial element in network security. The question that arises is which IDS is effective for our system? The answer should inevitably take into account the evaluation of IDSs effectiveness. Dealing with this challenge, many valuable evaluation metrics have been introduced such as receiver operating characteristic (ROC) curve, Bayesian detection rate, intrusion detection capability, intrusion detection operating characteristic, cost-based metrics, etc. The benefits and drawbacks of these metrics are discussed in this paper. We subsequently propose a novel metric called intrusion detection effectiveness $\left(E_{I D}\right)$ that manipulates the drawbacks of the existing ones, taking into account all essential and related parameters. We demonstrate the utility of $E_{I D}$ over the previously proposed ones, and how it realizes the measurement of the actual effectiveness rather than the relative effectiveness as followed by the existing ones. $E_{I D}$ can be used for evaluating the wired or wireless IDSs effectiveness. Additionally, we conduct experimental evaluation of two popular wireless IDSs (WIDSs), Kismet and AirSnare, to illustrate the benefits of $E_{I D}$.
\end{abstract}

Keywords: IDSs effectiveness, evaluation metrics, intrusion detection, false alarms.

\section{$1 \quad$ Introduction}

Despite the importance of intrusion detection systems (IDSs) in network security, their performance is sometimes not satisfying in practice. Thus, evaluating the IDSs performance is a pressing necessity. Many attributes judge the IDSs performance such as effectiveness, efficiency, interoperability [1], redundant alerts correlation, attack type recognizing, the impact on the supervised system resources, scalability and flexibility, etc. No doubt that the IDSs effectiveness is considered the main attribute and basic factor in evaluating the IDSs performance, where it reflects the ability of the IDS to detect the intrusive activities and the absence degree of the false alarms; they are considered the main great challenges facing the IDSs performance.

Evaluation metrics play the significant role in measuring and evaluating the IDSs effectiveness. In this paper, we study the well-known existing metrics for the IDSs effectiveness evaluation, such as receiver operating characteristic (ROC) [2] [3], Bayesian detection rate $(P(I \mid A))[1]$, cumulative cost [4], expected cost [5], intrusion 
detection capability $\left(C_{I D}\right)$ [6], and intrusion detection operating characteristic $(I D O C)$ [7]. Each of these metrics is based on a different theoretical approach such as decision theory [5], information theory [6], cost-based analysis [4] [5], etc. The strengths and weaknesses of these metrics are discussed in this paper, and consequently we propose a novel evaluation metric called intrusion detection effectiveness $\left(E_{I D}\right)$ that manipulates the drawbacks of the existing ones, especially the common main drawback that is manifested in their main notion of measuring the IDSs effectiveness on the basis of comparing two IDSs or more to select the best one, whereas this selected one may be ineffective.

Our developed metric $E_{I D}$ helps in measuring the actual effectiveness of IDSs rather than measuring the relative effectiveness as followed by the previously proposed metrics. The notion of $E_{I D}$ is based on comparing the operation curve of the IDS under test to the optimal operation curve (i.e., created as a zero reference curve for the optimal operation state) by calculating the variation between the two curves. The variation value interprets the deviation of the IDS operation from the intended optimal operation.

The rest of this paper is organized as follows. Section 2 studies the existing metrics, their benefits, and their drawbacks. Section 3 introduces the novel proposed metric $E_{I D}$ for evaluating the IDSs effectiveness. Section 4 presents the proof of the concept to achieve a credible evaluation of two popular WIDSs (Kismet and AirSnare). Finally, section 5 presents our conclusion and perspective.

\section{Related Work}

Various appreciable efforts have been exerted in the recent past for developing reliable evaluation metrics. In this section, we are concerned with analyzing the most valuable and well-known metrics for evaluating the IDSs effectiveness. Bayesian detection rate has a great concern in this paper, where we manipulate it to extract the base equation for our proposed metric $E_{I D}$ (intrusion detection effectiveness).

Receiver Operating Characteristic (ROC). The first unified metric used in the experimental evaluation of IDSs is the receiver operating characteristic $(R O C)$ curve, as applied by DARPA evaluations [2] [3]. ROC curve is used to analyze the trade-off between detection rate and false alarms rate. The notion of using $R O C$ curve in IDSs evaluation is based on comparing the IDSs curves to select the best one. If $R O C$ curves don't cross, then the upper curve with the higher values of detection rate is considered better than the lower ones. But, if $R O C$ curves are crossed, then the differentiation between them is based on the area under each curve. One of the drawbacks of $R O C$ curve is its disregard of base-rate parameter [1] that is considered a significant parameter in the IDSs evaluation, where it reflects the hostility of the operating environment (i.e., represented by the prior probability of intrusion).

Bayesian Detection Rate $(\boldsymbol{P}(\boldsymbol{I} \mid \boldsymbol{A}))$. Bayesian detection rate [1] defines a mathematical relation between the main parameters related to the intrusion detection effectiveness, i.e., detection rate, false alarms rate, and base-rate. The main 
advantage of this metric is its consideration of the base-rate parameter or probability of intrusion $(P(I))$. Bayesian detection rate $(P(I \mid A))$ (Eq. 1) was mainly derived from the Bayes' theorem by considering the possible events related to IDSs.

$$
P(I \mid A)=\frac{P(I) \cdot P(A \mid I)}{P(I) \cdot P(A \mid I)+P(\neg I) \cdot P(A \mid \neg I)}
$$

Where $I, \neg I, A, P(A \mid I)$, and $P(A \mid \neg I)$ denote intrusions, normal traffic, alarms, detection rate, and false alarms rate respectively. Axelsson in [1] studied the effect of the base-rate fallacy on the intrusion detection, and he demonstrated that the limiting factor for IDS performance is not the ability to correctly identify the intrusions, but rather its ability to suppress the false alarms. We totally agree with this conclusion.

Unfortunately, despite the prominence of Bayesian detection rate and its consideration of the significant parameters for the IDSs effectiveness evaluation, it is not completely expressive metric for measuring the IDSs effectiveness. We analyze $P(I \mid A)$ (Eq. 1) mathematically to reach the following results:

Case 1: if $P(A \mid I)=P(A \mid \neg I)=1$

Combining these values with equation Eq. 1, then;

$$
P(I \mid A)=\frac{P(I)}{P(I)+P(\neg I)}
$$

Since $P(I)+P(\neg I)=1$, then;

$$
P(I \mid A)=P(I)
$$

Case 2: if $P(A \mid I)=0$

Combining this value and equation Eq. 1, then;

$$
P(I \mid A)=0
$$

$P(I \mid A)$ gives reasonable expressions for the IDSs effectiveness, just in the above two cases. In case 1 of passing all the traffic with raised alarms $(P(A \mid I)=$ $P(A \mid \neg I)=1), P(I \mid A)$ equals $P(I)$ (Eq. 2 ) that is considered the perfect expression in this case; where the ratio of the detected intrusions to the generated alarms corresponds to the ratio of the intrusions to the input traffic. In case 2, $P(I \mid A)$ equals zero when the detection rate $P(A \mid I)$ comes to nought (Eq. 3), where $P(A \mid I)$ is the predominant parameter in equation Eq. 1. However, the drawback of Bayesian detection rate $P(I \mid A)$ is manifested when the false alarms or false positive rate $P(A \mid \neg I)$ is equal or close to zero as shown in the following case 3 .

Case 3: as $P(A \mid \neg I)$ approaches 0, then equation Eq. 1 can be written as;

$$
\lim _{P(A \mid \neg I) \rightarrow 0} P(I \mid A)=\lim _{P(A \mid \neg I) \rightarrow 0} \frac{P(I) \cdot P(A \mid I)}{P(I) \cdot P(A \mid I)+P(\neg I) \cdot P(A \mid \neg I)}=1
$$

As we observe from equation Eq. 4, when $P(A \mid \neg I)$ equals or approaches " 0 ", $P(I \mid A)$ is equal to constant value " 1 " for any value of $P(A \mid I)$; this unfortunately seems illogical. How the IDSs effectiveness can be evaluated in disregard of the detection rate $P(A \mid I)$ ? Merely considering the complete absence of false alarms is 
insufficient. Thus $P(I \mid A)$, in this case, is inexpressive metric for the IDSs effectiveness or even the detection rate. However, we propose a reasonable solution for this drawback through our manipulation of $P(I \mid A)$ to become completely expressive in a new form called enhanced Bayesian detection rate $(E B D)$ (section 3.1). We use $E B D$ as a base equation for our proposed metric $E_{I D}$ (section 3.2).

Cost-Based Metrics. Cost-based metrics analyze the intrusion detection from the perspective of costs. Stolfo et al. [4] proposed cumulative cost metric for evaluating the fraud and intrusion detection in the financial information systems. They defined three types of costs; operational, damage, and challenge costs. Operational cost refers to the resources needed to run the IDS. Damage cost is the amount of damage caused by the leaked undetected intrusions. Challenge cost is the cost of acting upon an intrusion when it is detected. CumulativeCost $(S)$ metric (Eq. 5) is derived by considering the challenge and damage costs on the outcome events of IDSs plus the operational cost to evaluate the IDSs over test set " $S$ " of labeled connection " $c$ ". One of the drawbacks of this metric is its disregard of the base-rate parameter.

$$
\text { CumulativeCost }(S)=\sum_{c \in S}(\operatorname{Cost}(c)+O p \operatorname{Cost}(c))
$$

The second noted metric is expected cost metric that was proposed by Gaffney and Ulvila [5] who argued that both ROC analysis and cumulative cost metric are incomplete metrics. They used decision analysis techniques to combine and extend $R O C$ analysis and cost-based analysis to provide expected cost metric that considers the base-rate parameter. More details about this metric are available in [5].

Intrusion Detection Capability $\left(\boldsymbol{C}_{I D}\right)$. $\mathrm{Gu}$ et al. [6] proposed an informationtheoretic measure of the intrusion detection capability. They depended on the notion of having less uncertainty about the IDS input, given the IDS output. They introduced $C_{I D}$ metric (Eq. 6) as the mutual information between the IDS input $X$ and output $Y$ normalized by the entropy (or self-information) of the input $H(X) . C_{I D}$ is maximized by calculating the operating point that minimizes the uncertainty of the input.

$$
C_{I D}=\frac{I(X ; Y)}{H(X)}=\frac{H(X)-H(X \mid Y)}{H(X)}
$$

Where $H(X \mid Y)$ is the entropy of $X$ given $Y$. We believe that the notion of $C_{I D}$ for minimizing the uncertainty of the input is inapplicable in the IDSs evaluation domain.

Intrusion Detection Operating Characteristic (IDOC). Cardenas et al. [7] used $P(I \mid A)$ (Eq. 1) and introduced the intrusion detection operating characteristic $(I D O C)$ as a trade-off curve between the $P(I \mid A)$ and the probability of intrusion detection $P(A \mid I)$. As a consequence of the dependence of IDOC on the Bayesian detection rate equation, it carries all its drawbacks.

The common drawback of most existing metrics lies in their main notion of comparing two or more IDSs to select the best one, although this selected one may be ineffective. This is considered a deficient approach that leads to measuring the relative effectiveness rather than the actual effectiveness. We are concerned with manipulating this drawback and the above mentioned ones. 


\section{Intrusion Detection Effectiveness $\left(E_{I D}\right)$}

The logical approach for measuring the actual effectiveness is comparing the IDS under test to the optimal operation level (as a reference). We thus propose a new evaluation metric $E_{I D}$ (intrusion detection effectiveness) that is based on the notion of comparing the operation curve of the IDS under test to the optimal operation curve (created as a zero reference curve) by calculating the variation between the two curves. The variation value interprets the deviation of the IDS from the intended optimal operation. We believe that the main parameters which the IDS effectiveness depends on are detection rate, false alarms rate, and base-rate. To realize the notion of $E_{I D}$, we need an expressive formula or equation that considers these parameters to be used as a base for $E_{I D}$. As a result of our research, we discovered that Bayesian detection rate (Eq. 1) regards the needed parameters, but it is inappropriate as a base equation due to its drawback when the false alarms rate equals or approaches "0" (Eq. 4). Consequently, we manipulate this drawback to derive a new completely expressive formula called enhanced Bayesian detection rate $(E B D)$ to become the base for $E_{I D}$.

\subsection{Deriving the Enhanced Bayesian Detection Rate (EBD)}

As a brief summary of our analysis of Bayesian detection rate $P(I \mid A)$ (section 2), in case $1(P(A \mid I)=P(A \mid \neg I)=1)$ and case $2(P(A \mid I)=0), P(I \mid A)$ gives reasonable expressions, but it is inexpressive in case 3 (as $P(A \mid \neg I$ ) equals or approaches " 0 "). Accordingly, we are concerned with manipulating case 3. By analyzing case 3 , we conclude that the logical expressive formula for $P(I \mid A)$, as $P(A \mid \neg I)$ equals or approaches " 0 ", should be equal to $P(A \mid I)$. This can be achieved by modifying the denominator of equation Eq. 4 to produce the following new formula.

$$
\begin{aligned}
\lim _{P(A \mid \neg I) \rightarrow 0} P(I \mid A) & =\lim _{P(A \mid \neg I) \rightarrow 0} \frac{P(I) \cdot P(A \mid I)}{P(I) \cdot P(A \mid I)_{\uparrow=1}+P(\neg I) \cdot P(A \mid \neg I)} \\
& =\frac{P(I) \cdot P(A \mid I)}{P(I) \cdot P(A \mid I)_{\curvearrowright=1}}=P(A \mid I)
\end{aligned}
$$

From equations Eq. 1, Eq. 2, Eq. 3 and Eq. 7 we produce the enhanced Bayesian detection rate $(E B D)$ (Eq. 8) that is completely expressive under all operation conditions.

$$
E B D=\frac{P(I) \cdot P(A \mid I)}{P(I)+P(\neg I) \cdot P(A \mid \neg I)}
$$

\section{Property 1}

$E B D$ can be defined as the posterior probability of the detected intrusions $(T P)$ given the total output of intrusion related responses $(T P+F N)$ and false alarms $(F P)$.

Proof

The intrusion detection can be summarized by the simple model shown in Fig. 1, where $I, \neg I, A, \neg A, T P, F P, F N$, and $T N$ denote intrusions, normal traffic, alarms, no alarms, true positives, false positives, false negatives, and true negatives respectively. 


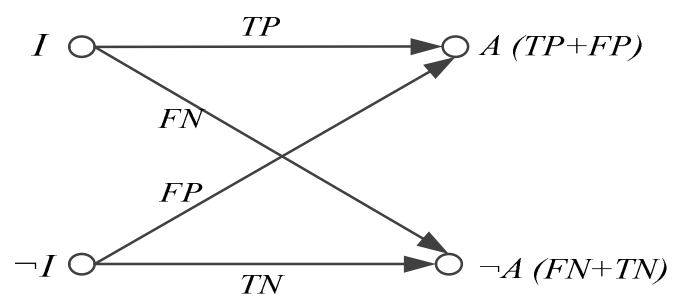

Fig. 1. Intrusion Detection Model

Basically,

$$
\begin{aligned}
& P(I)=I /(\neg I+I) \\
& P(\neg I)=\neg I /(\neg I+I) \\
& P(A \mid I)=T P / I=T P /(T P+F N) \\
& P(A \mid \neg I)=F P / \neg I=F P /(F P+T N)
\end{aligned}
$$

Recalling equation Eq. 8 and solving it by these parameters, then;

$$
\begin{gathered}
E B D=\frac{P(I) \cdot P(A \mid I)}{P(I)+P(\neg I) \cdot P(A \mid \neg I)}=\frac{1}{\frac{1}{P(A \mid I)}+\frac{P(\neg I) \cdot P(A \mid \neg I)}{P(I) \cdot P(A \mid I)}} \\
=\frac{1}{\frac{T P+F N}{T P}+\frac{\neg I \cdot F P /(F P+T N)}{\ddagger \cdot T P /(P P}}=\frac{T P}{T P+F N+F P}
\end{gathered}
$$

Equation Eq. 9 shows the significance of $E B D$ for measuring the proportion of the intrusion related responses $(T P+F N)$ and false alarms $(F P)$ that correspond to the detected intrusions (TP). This is considered one of the great advantages of $E B D$ over $P(I \mid A)$ that ignores the false negatives $(F N)$, as demonstrated in the following.

By recalling $P(I \mid A)$ (Eq. 1) and solving it in the same way, then;

$$
\begin{gathered}
P(I \mid A)=\frac{P(I) \cdot P(A \mid I)}{P(I) \cdot P(A \mid I)+P(\neg I) \cdot P(A \mid \neg I)}=\frac{1}{1+\frac{P(\neg I) \cdot P(A \mid \neg I)}{P(I) \cdot P(A \mid I)}} \\
=\frac{1}{1+\frac{\neg I \cdot F P /(F P+T N)}{\ddagger \cdot T P /(T P+F N)}}=\frac{T P}{T P+F P}
\end{gathered}
$$

Equation Eq. 10 clarifies that $P(I \mid A)$ disregards $F N$ parameter that influences the expressiveness of the metric.

To clarify more the benefit of $E B D$ over $P(I \mid A)$ regarding taking the whole false responses $(F P+F N)$ into account, the relationships between the IDS input and output events are depicted through Venn diagram (Fig. 2). The intersections between the different events are represented by the areas denoted by numbers from 1 to 4 . Area 1 represents the tranquil area of no intrusion and no alarm, but areas 2, 3 and 4 represent the challenge areas of false responses (areas 2 and 4) and detected intrusions (area 3). These events in the areas 2, 3 and 4 have a great significance in the IDSs effectiveness evaluation, and they should be considered by the evaluation metric. This is attained by $E B D$ as shown in equation Eq. 9. On the contrary, $P(I \mid A)$ considers only the events of the areas 3 and 4 as shown by Eq. 10 . 


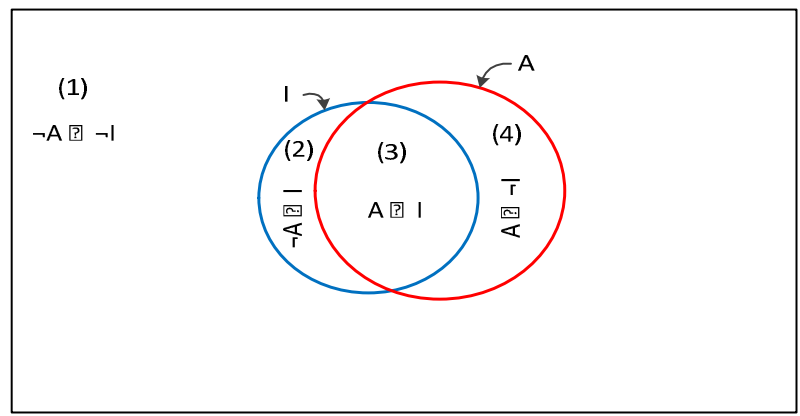

Fig. 2. The Relationships between the IDS Input and Output Events

\section{Property 2}

$E B D$ is an expressive metric under different operation conditions.

\section{Proof}

$E B D$ agrees with $P(I \mid A)$ on the aforementioned case 1 (Eq. 2) and case 2 (Eq.3), and gives the same expressive results. As well, $E B D$ gives an expressive value in the third case (Eq. 7) according to its mathematical manipulation. For the other rest conditions, $E B D$ can be analyzed as follows.

Case 4: if $P(A \mid \neg I)=1$

Combining this value with equation Eq. 8, then;

$$
E B D=\frac{P(I) \cdot P(A \mid I)}{P(I)+P(\neg I)}
$$

Since $P(I)+P(\neg I)=1$, then;

$$
E B D=P(I) \cdot P(A \mid I)=\frac{t}{(\neg I+I)} \cdot \frac{T P}{t}=\frac{T P}{T P+F N+F P}
$$

Equation Eq. 11 demonstrates the expressiveness of $E B D$ in the worst case of false alarms $(P(A \mid \neg I)=1)$.

Case 5: if $P(A \mid I)=1$

Combining this value with equation Eq. 8, then;

$$
E B D=\frac{P(I)}{P(I)+P(\neg I) \cdot P(A \mid \neg I)}=\frac{1}{1+\frac{\neg I \cdot F P /(F P+T N)}{T P}}=\frac{T P}{T P+F P}
$$

As shown in equation Eq. 12, EBD equals the proportion of the generated alarms that correspond to the detected intrusions. This is the expressive formula in this case of the absence of false negatives $(P(A \mid I)=1 \Leftrightarrow F N=0)$. The above analysis concludes the expressiveness of $E B D$ under different operation conditions. 


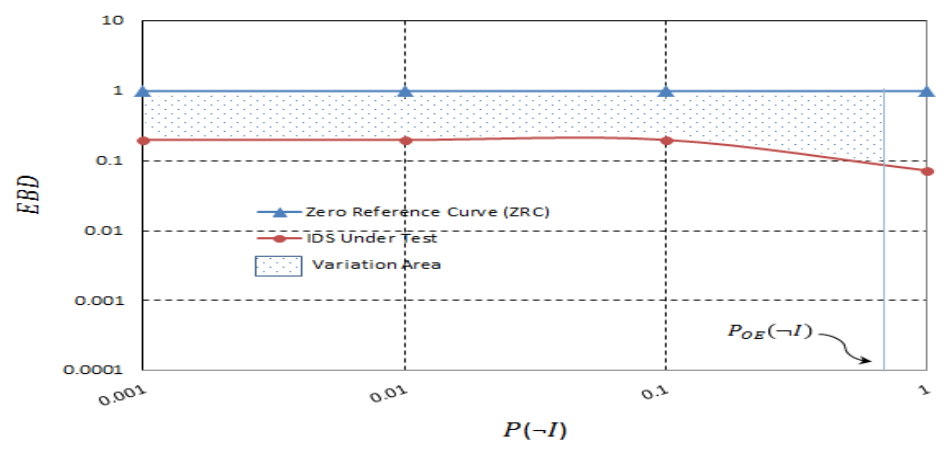

Fig. 3. The trade-off between $E B D$ and $P(\neg I)$

\subsection{Deriving the Intrusion Detection Effectiveness $\left(E_{I D}\right)$}

Following the main notion of our metric $E_{I D}$, we consider the trade-off between $E B D$ and $P(\neg I)$ that helps in developing the expressive metric $E_{I D}$. To simplify dealing with $E B D$ (Eq. 8), we adapt it to be a function of $P(\neg I)$ as shown in Eq. 13.

$$
E B D=\frac{P(A \mid I)-P(\neg I) \cdot P(A \mid I)}{1-P(\neg I) \cdot(1-P(A \mid \neg I))}
$$

The first step in deriving $E_{I D}$ is calculating and plotting the zero reference curve $(Z R C)$ as a trade-off between $E B D$ and $P(\neg I)$ with assumption of the optimal operation case of the IDS under test. To clarify the idea of calculating and plotting $Z R C$, we assume an IDS installed in an operating environment with hostility or probability of intrusion $P(I)=3 * 10^{-4}$. Then, the probability of no intrusion $P(\neg I)=$ $1-P(I)=0.9997$. First, we assume that the IDS under test operates at the optimal case with perfect detection rate $(P(A \mid I)=1)$ and complete absence of false alarms $(P(A \mid \neg I)=0)$. We combine these values with $E B D$ (Eq. 13) to plot $Z R C$ (Fig. 3). As a note, the axes are set to logarithmic scale. The second step, we plot the real operation curve of the IDS with the actual values of the detection rate and false alarms; we assume their values as $P(A \mid I)=0.2$ and $P(A \mid \neg I)=0.0035$. Now we have two operation curves; one as a $Z R C$ curve for the optimal operation and the other represents the actual operation curve (Fig. 3). The variation between the two curves is represented by the dotted area. $P_{O E}(\neg I)$ denotes the probability of no intrusion in the operating environment, and it refers to the upper limit of the variation area.

We normalize this variation by the area under $Z R C$ (only through $P(\neg I)=[0$, $\left.\left.P_{O E}(\neg I)\right]\right)$ to have a representative metric $E_{I D}$ of values in the range $[0,1]$; where " 0 " indicates zero deviation from the intended optimal operation and then perfect effectiveness, but " 1 " indicates the maximum deviation and then zero effectiveness. $E_{I D}$ is represented by equation Eq. 14, where $E B D_{Z R C}, P_{Z R C}(A \mid I)$, and $P_{Z R C}(A \mid \neg I)$ denote $E B D$, detection rate, and false alarms of $Z R C$ respectively. As well, $E B D_{I D}$, $P_{I D}(A \mid I)$, and $P_{I D}(A \mid \neg I)$ denote $E B D$, detection rate, and false alarms of IDS under test respectively. $P(\neg I)$ is considered the integration variable. 


$$
\begin{gathered}
E_{I D}=\frac{1}{\int_{0}^{P_{O E}(\neg I)} E B D_{Z R C} d P(\neg I)}\left(\int_{0}^{P_{O E}(\neg I)} E B D_{Z R C} d P(\neg I)\right. \\
\left.-\int_{0}^{P_{O E}(\neg I)} E B D_{I D} d P(\neg I)\right)
\end{gathered}
$$

Where,

$$
\begin{aligned}
& E B D_{Z R C}=\frac{P(I) \cdot P_{Z R C}(A \mid I)_{\neg=1}}{P(I)+P(\neg I) \cdot P_{Z R C}(A \mid \neg I)_{\neg=0}}=1 \\
& E B D_{I D}=\frac{P_{I D}(A \mid I)-P(\neg I) \cdot P_{I D}(A \mid I)}{1-P(\neg I) \cdot\left(1-P_{I D}(A \mid \neg I)\right)}
\end{aligned}
$$

Then equation Eq. 14 becomes;

$$
E_{I D}=1-\frac{\int_{0}^{P_{O E}(\neg I)} E B D_{I D} d P(\neg I)}{P_{O E}(\neg I)}
$$

Equation Eq. 17 can be solved mathematically [8], and $E_{I D}$ becomes;

$$
E_{I D}=1-\frac{P_{I D}(A \mid I) \cdot\left(P_{O E}(\neg I)+\left(\frac{1}{\left(1-P_{I D}(A \mid \neg I)\right)}-1\right) \ln \left|1-P_{O E}(\neg I) \cdot\left(1-P_{I D}(A \mid \neg I)\right)\right|\right)}{P_{O E}(\neg I) \cdot\left(1-P_{I D}(A \mid \neg I)\right)}
$$

\section{Property 3}

$E_{I D}$ is an expressive metric for measuring the actual effectiveness of IDSs by values in the range $[0,1]$, where " 0 " indicates the ideal case of supreme effectiveness, but " 1 " indicates the worst case of zero effectiveness.

\section{$\underline{\text { Proof }}$}

Case 1: when the IDS detects all the intrusive activities $(P(A \mid I)=1)$ and generates no false alarms $(P(A \mid \neg I)=0)$, then its deviation from the optimal operation case can be measured by $E_{I D}$ (Eq. 18) as follows.

$$
E_{I D}=1-\frac{P_{I D}(A \mid I)_{\rightarrow=1} \cdot\left(P_{O E}(\neg I)+0\right)}{P_{O E}(\neg I) \cdot\left(1-P_{I D}(A \mid \neg I)_{\uparrow=0}\right)}=1-\frac{P_{\theta_{E}}(\neg I)}{P_{O E}(\neg I)}=0
$$

Equation Eq. 19 demonstrates the supreme effectiveness of the IDS by its zero deviation from the optimal operation case.

Case 2: when the IDS fails to detect the intrusions $(P(A \mid I)=0)$, its deviation from the optimal operation case can be measured by $E_{I D}$ (Eq. 18) as follows.

$$
E_{I D}=1-\frac{0}{P_{O E}(\neg I) \cdot\left(1-P_{I D}(A \mid \neg I)\right)}=1
$$

Equation Eq. 20 demonstrates the maximum deviation of the IDS from the optimal operation case and accordingly its ineffectiveness.

Besides property 1 and property 2 of $E B D$ that is the base equation of $E_{I D}$, it becomes clear from equations Eq. 19 and Eq. 20 that $E_{I D}$ is an expressive metric for measuring the actual effectiveness. $E_{I D}$ can be used for evaluating the effectiveness of wired or wireless IDSs. 


\section{Proof of the Concept}

As a proof of the concept, we conduct an experimental evaluation of two popular wireless IDSs (WIDSs) Kismet (for Linux) [9] and AirSnare (for Windows) [10], and measure their effectiveness using $E_{I D}$. We used RF shielded testbed, an access point Linksys WRT54GL, and workstations (Linux and Windows) with Wi-Fi adapters ALFA awus036h and D-Link DWA-110 with respect to the compatibility with the operating systems and the WIDSs. We resorted to use the RF shielded testbed to circumvent the problem of the uncontrolled 802.11 traffic from the adjacent wireless stations that obstructs the accurate measurements of the considered parameters. We are concerned with the wireless infrastructure mode with two possible scenarios for the installation of WIDSs (Fig. 4); scenario 1: the WIDS was installed on the access point, and scenario 2: the WIDS was installed on a terminal machine as a victim. As for the normal background traffic, we generated real traffic by capturing the operational traffic during the normal operation of a private network installed for this purpose, and then replaying the collected traffic into the testbed. This private network (Fig. 4) consists of an access point, three workstations (i.e., two machines operate under Windows and the third one operates under Linux) and two mobile phones (i.e., Android system). Table 1 shows the statistics of the collected benign traffic.

As for the generated attacks, the credible evaluation of WIDSs necessitates taking into account all possible attacks. While this is operationally impossible, it is necessary to select representative attack test cases that are extracted mainly from a holistic classification of wireless attacks. Dealing with this challenge, we used our developed taxonomy of wireless attacks from the perspective of the WIDSs evaluator [11] and we generated the attacks listed in Table 2 according to the representative attack test cases shown in Fig. 5. As well, the attack detection of each WIDS is shown in Table 2. For calculating the detection rate, if we follow the ordinary method that was used in the previous evaluations of IDSs, then Table 2 is sufficient for the calculations and then the detection rate is $P(A \mid I)=0.61$ for Kismet, and $P(A \mid I)=0.167$ for AirSnare. These values are not real expressive values for the detection rate, and subsequently have a negative effect on the calculation of the real effectiveness. The best way for calculating the expressive detection rate is considering the probability of occurrence of the attack test cases under the operating environment conditions.
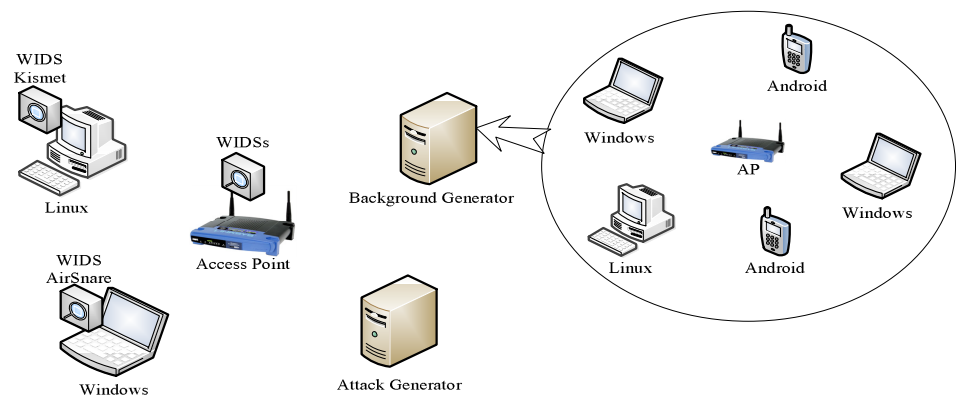

Fig. 4. Evaluation Testbed 
Table 1. The Collected Normal Traffic

\begin{tabular}{|l|c|}
\hline \multicolumn{1}{|c|}{ Frame Subtype } & Frame Count \\
\hline Association request & 38 \\
\hline Association response & 43 \\
\hline Reassociation request & 172 \\
\hline Reassociation response & 142 \\
\hline Probe request & 227081 \\
\hline Probe response & 218602 \\
\hline Beacon & 378 \\
\hline Disassociation & 332 \\
\hline Authentication & 169 \\
\hline Deauthentication & 80 \\
\hline Action frames & 3484 \\
\hline Null data & 89920 \\
\hline QoS data & 1723 \\
\hline QoS null data & 19868 \\
\hline Total & 562032 \\
\hline
\end{tabular}

Table 2. The Attack Detection

\begin{tabular}{|l|c|c|}
\hline \multirow{2}{*}{ Generated Attacks } & Kismet & AirSnare \\
\cline { 2 - 3 } & $T P$ & $T P$ \\
\hline Deauthentication/Disassociation Flood (<10 Request) & $\checkmark$ & $\mathrm{x}$ \\
\hline Deauthentication/Disassociation Flood (<20 Request) & $\checkmark$ & $\mathrm{x}$ \\
\hline Deauthentication/Disassociation Flood (>30 Request) & $\checkmark$ & $\checkmark$ \\
\hline Deauthentication/Disassociation Flood (>100 Request) & $\checkmark$ & $\checkmark$ \\
\hline Deauthentication/Disassociation (Amok mode) & $\checkmark$ & $\mathrm{x}$ \\
\hline Fake Authentication & $\checkmark$ & $\mathrm{x}$ \\
\hline Authentication Flood & $\checkmark$ & $\mathrm{x}$ \\
\hline Beacon Flood (evil duplicate AP DoS) & $\checkmark$ & $\mathrm{x}$ \\
\hline MITM attack & $\mathrm{x}$ & $\mathrm{x}$ \\
\hline ARP Request Replay Attack & $\mathrm{x}$ & $\mathrm{x}$ \\
\hline WPA Downgrade & $\checkmark$ & $\mathrm{x}$ \\
\hline WPA Cracking & $\checkmark$ & $\mathrm{x}$ \\
\hline WEP Cracking & $\mathrm{x}$ & $\mathrm{x}$ \\
\hline Chopchop & $\mathrm{x}$ & $\mathrm{x}$ \\
\hline Hidden SSID Brute Force & $\mathrm{x}$ & $\mathrm{x}$ \\
\hline Rogue AP & $\mathrm{x}$ & $\mathrm{x}$ \\
\hline RF Jamming & $\checkmark$ & $\mathrm{x}$ \\
\hline MAC Spoofing & $\mathrm{x}$ & $\checkmark$ \\
\hline
\end{tabular}

In our evaluation tests, we considered and used 100 attack instances of the attacks listed in Table 2. We considered the instances of the generated attacks by ratios that correspond approximately to the probability of attack occurrence in some real systems. This consideration was managed according to our statistical analysis of the registered wireless attacks and vulnerabilities in the popular database such as Common Vulnerabilities and Exposures [12], National Vulnerability Database - NIST [13], 


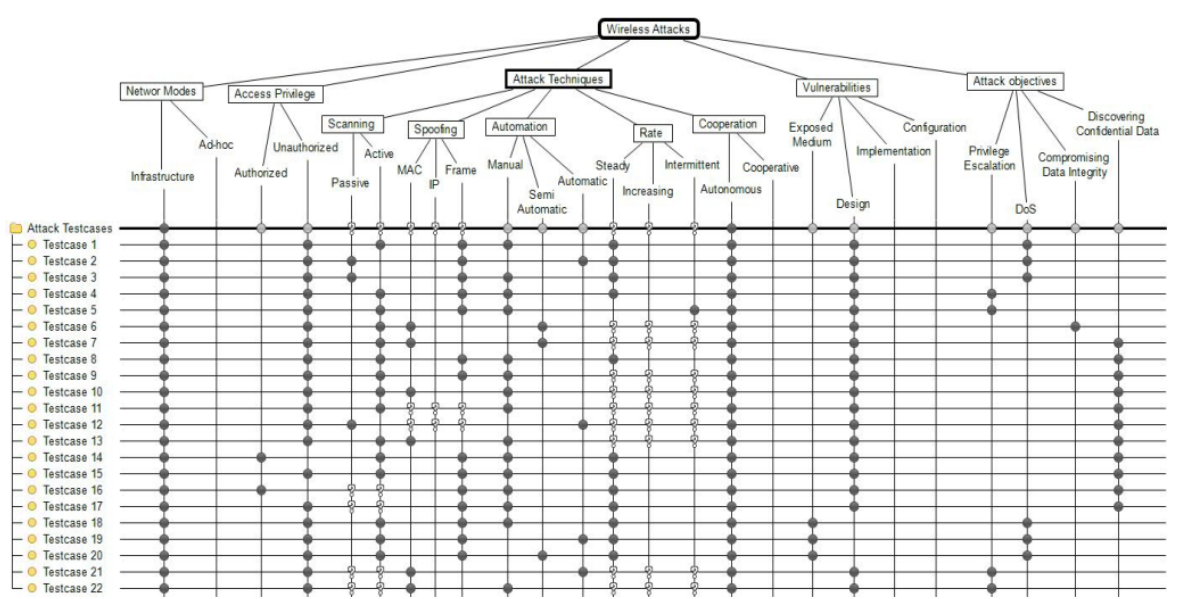

Fig. 5. Representative Attack Test Cases

Table 3. The Generated Attacks and the Corresponding Test Cases

\begin{tabular}{|l|c|}
\hline \multicolumn{1}{|c|}{ Generated Attacks } & Representative Attack Test Cases \\
\hline Deauthentication/Disassociation Flood & $1,2,3$ \\
\hline Deauthentication/Disassociation (Amok mode) & 3 \\
\hline Fake Authentication & 4,5 \\
\hline Authentication Flood & 2,3 \\
\hline Beacon Flood (evil duplicate AP DoS) & 3 \\
\hline MITM attack & 6,7 \\
\hline ARP Request Replay Attack & 8 \\
\hline WPA Downgrade & 3 \\
\hline WPA Cracking & 9 \\
\hline WEP Cracking & $10,11,12$ \\
\hline Chopchop & 13 \\
\hline Hidden SSID Brute Force & 11 \\
\hline Rogue AP & $14,15,16,17$ \\
\hline RF Jamming & $18,19,20$ \\
\hline MAC Spoofing & 21,22 \\
\hline
\end{tabular}

and others. It is worth mentioning that we considered in our calculations the deauthentication/disassociation flood attack instances with deauthentication requests $>30$ (Table 2); we generated it by 8 instances from total of 100 instances of all the generated attacks. We classified the generated attacks under the representative test cases (Fig. 5 and Table 3), and adjusted the estimated probability of occurrence as shown in Table 4. Then, the expressive detection rate is $P(A \mid I)=0.65$ for Kismet, and $P(A \mid I)=0.13$ for AirSnare. In our evaluation environment, the used 100 attack instances generated approximately 1500 malicious frames, in addition to the generated background normal traffic (Table 1). Then, we have hostility or intrusion probability $P(I)=1500 / 562032=2.66889^{*} 10^{-3}$, and no intrusion probability $P(\neg I)=0.99733$. As well, the registered false alarms for the two WIDSs are $P(A \mid \neg I)=0.008967$ for Kismet 
Table 4. Probability of Occurrence of the Generated Attack Instances

\begin{tabular}{|c|c|c|c|}
\hline \multirow{2}{*}{ Attack Test Cases } & \multirow{2}{*}{ Estimated Probability } & \multicolumn{2}{|c|}{ WIDSs Detection Ratio } \\
\cline { 3 - 4 } & & Kismet & AirSnare \\
\hline $1,2,3,4,5$ & 0.46 & $\checkmark$ & $\checkmark(0.08)$ \\
\hline 6,7 & 0.05 & $\mathrm{x}$ & $\mathrm{x}$ \\
\hline 8 & 0.04 & $\mathrm{x}$ & $\mathrm{x}$ \\
\hline 9 & 0.13 & $\mathrm{x}$ & $\mathrm{x}$ \\
\hline $10,11,12$ & 0.1 & $\mathrm{x}$ & $\mathrm{x}$ \\
\hline 13 & 0.04 & $\mathrm{x}$ & $\mathrm{x}$ \\
\hline $14,15,16,17$ & 0.07 & $\checkmark$ & $\mathrm{x}$ \\
\hline $18,19,20$ & 0.06 & $\mathrm{x}$ & $\checkmark$ \\
\hline 21,22 & 0.05 & 0.65 & 0.13 \\
\hline Total & 1 & & \\
\hline
\end{tabular}

and $P(A \mid \neg I)=0.0014946$ for AirSnare. Combining these obtained results with our proposed evaluation metric $E_{I D}$ (Eq. 18), then;

$$
\begin{aligned}
& E_{I D}(\text { kismet })=0.37 \\
& E_{I D}(\text { AirSnare })=0.871
\end{aligned}
$$

In the same way, as described in section 3.2, we can plot the operation curves of the two WIDSs, besides the zero reference curve $(Z R C)$ as shown in Fig. 6. $E_{I D}$ of the two WIDSs and Fig. 6 show that Kismet operation doesn't deviate much more from the optimal case $Z R C$, in contrast to AirSnare that has a great deviation from the optimal case. Then, Kismet is more effective than AirSnare.

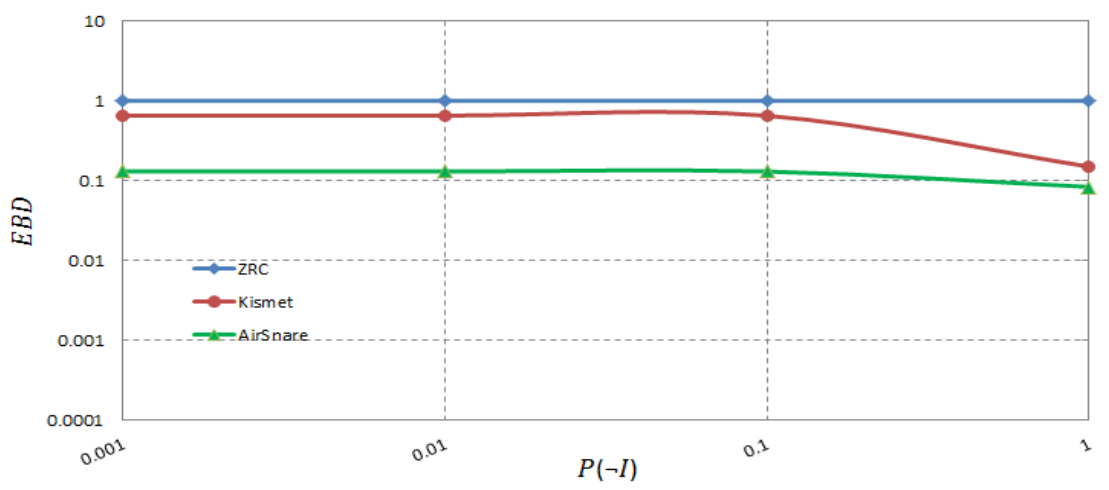

Fig. 6. The Trade-off between $E B D$ and $P(\neg I)$ of Kismet, AirSnare, and $Z R C$

\section{Conclusion}

Our proposed metric $E_{I D}$ manipulated the drawbacks of the existing metrics and it realizes the measurement of the actual effectiveness, taking into account the main related parameters. We conducted credible evaluation of two popular WIDSs (Kismet and AirSnare) using $E_{I D}$ and considered some important aspects that were ignored in 
the existing work such as the probability of occurrence of attacks and selecting the attacks on the basis of representative attack test cases. The results demonstrated that Kismet is more effective than AirSnare. We are interested in deriving other evaluation metrics for the rest attributes of IDSs/WIDSs performance.

\section{References}

1. Axelsson, S.: The Base-Rate Fallacy and its Implications for the Difficulty of Intrusion Detection. In: Proceedings of the 6th ACM Conference on Computer and Communications Security (CCS 1999), pp. 1-7. ACM Press (1999)

2. Lippmann, R., Fried, D., Graf, I., Haines, J., Kendall, K., McClung, D., Weber, D., Webster, S., Wyschogrod, D., Cunningham, R., Zissman, M.: Evaluating Intrusion Detection Systems: The 1998 DARPA Off-line Intrusion Detection Evaluation. In: Proceedings of DARPA Information Survivability Conference and Exposition (DISCEX 2000), Los Alamitos, CA, USA, pp. 12-26 (2000)

3. Lippmann, R., Haines, J.W., Fried, D., Korba, J., Das, K.: Analysis and Results of the 1999 DARPA Off-Line Intrusion Detection Evaluation. In: Debar, H., Mé, L., Wu, S.F. (eds.) RAID 2000. LNCS, vol. 1907, pp. 162-182. Springer, Heidelberg (2000)

4. Stolfo, S., Fan, W., Lee, W., Prodromidis, A., Chan, P.: Cost-based modeling for fraud and intrusion detection: Results from the JAM project. In: Proceedings of DARPA Information Survivability Conference and Exposition (DISCEX 2000), pp. 130-144 (2000)

5. Gaffney, J.E., Ulvila, J.W.: Evaluation of intrusion detectors: A decision theory approach. In: Proceedings of IEEE Symposium on Security and Privacy (S\&P 2001), Oakland, CA, USA, pp. 50-61 (2001)

6. Gu, G., Fogla, P., Dagon, D., Lee, W., Skoric, B.: Measuring Intrusion Detection Capability: An Information-Theoretic Approach. In: Proceedings of ACM Symposium on InformAtion, Computer and Communications Security (ASIACCS 2006), Taipei, Taiwan (2006)

7. Cardenas, A.A., Baras, J.S., Seamon, K.: A Framework for the Evaluation of Intrusion Detection Systems. In: Proceedings of IEEE Symposium on Security and Privacy (S\&P 2006), Oakland, CA, USA, pp. 63-77 (2006)

8. Zill, D.G., Wright, W.S.: Advanced Engineering Mathematics. Jones \& Bartlett Learning (2012)

9. Kismet: Kismet-WIDS, http: / /www.kismetwireless.net/

10. AirSnare: AirSnare-WIDS, http://home. comcast. net/ jay. deboer/airsnare/

11. Nasr, K., Abou El Kalam, A., Fraboul, C.: An IDS evaluation-centric taxonomy of wireless security attacks. In: Wyld, D.C., Wozniak, M., Chaki, N., Meghanathan, N., Nagamalai, D. (eds.) CNSA 2011. CCIS, vol. 196, pp. 402-413. Springer, Heidelberg (2011)

12. CVE Wireless: Common Vulnerabilities and Exposures - Wireless, http://cve.mitre.org/cgi-bin/cvekey.cgi?keyword=wireless

13. NVD-NIST: National Vulnerability Database, National Institute of Standards and Technology (NIST), http: //nvd.nist.gov/home.cfm 